\title{
Investigation of Single Event Latch-up effects in the ALICE SAMPA ASIC
}

\section{Sohail Musa Mahmood"University of Oslo (NO)}

E-mail: s.m.mahmoodefys.uio.no

\section{Ketil Røed}

University of Oslo (NO)

E-mail: ketil.roedefys.uio.no

\section{for the ALICE Collaboration}

During RUN 3 and RUN 4 at the Large Hadron Collider (LHC), the SAMPA chip will be used in the upgraded front end electronics of the ALICE (A Large Ion Collider Experiment) Time Projection Chamber (TPC) and Muon Chambers (MCH). Previously, it was reported that the SAMPA V2 prototypes were susceptible to the high energy proton induced Single Event Latch-up (SEL) events. Further irradiation campaigns were required to find the source of SEL events in SAMPA V2 prototypes, and to verify that the SEL sensitivity of final versions (V3 and V4) of the SAMPA chip was reduced or even completely removed. The irradiation campaigns were performed using the Heavy-Ion Facility (HIF) at UCL (Universitè Catholique de Louvain) in Belgium and the Single-Photon laser facility at IES (Institute of Electronics and Systems), Montpellier-France.

Topical Workshop on Electronics for Particle Physics (TWEPP2018)

17-21 September 2018

Antwerp, Belgium

\footnotetext{
* Speaker.
} 


\section{Introduction}

The ALICE experiment is one of the four major experiments at LHC which studies particle production in heavy-ion $(\mathrm{Pb}-\mathrm{Pb}), \mathrm{p}-\mathrm{Pb}$ and $\mathrm{pp}$ collisions. After the second long shutdown (LS2) ending in 2021, the expected interaction rate for $\mathrm{Pb}-\mathrm{Pb}$ collisions in the ALICE experiment will be increased from $8 \mathrm{kHz}$ to $50 \mathrm{kHz}$ [1]. The present readout electronics of the Time Projection Chamber (TPC) and Muon Chambers $(\mathrm{MCH})$ are not capable of handling the higher collision rates. Thus, a new common custom-made readout chip named SAMPA has been designed to replace the current readout chips in both sub-detectors [1]. The SAMPA chip includes 32 data processing channels, each containing a charge-sensitive pre-amplifier (CSA), a pulse shaper, a 10-bit $10 \mathrm{MHz}$ Analog to Digital Converter (ADC), and a Digital Signal Processor (DSP) module [2]. For the data storage in the DSP, both the registers and the Static Random Access Memory (SRAM) IPs are implemented in the SAMPA chip.

High Energy Hadrons (HEH) are the primary source of radiation induced Single Event Effects (SEEs) through indirect ionization in the readout electronics [1]. SEEs are caused by the passage of a single energetic particle through the sensitive regions of the digital circuits, disrupting their correct operations. They are mainly classified into soft errors (Single Event Upset, Single Event Transient) and hard errors (Single Event Latchup). SEEs are the primary concern for the SAMPA digital design as the expected HEH flux for the SAMPA chip will increase up to $3.4 \mathrm{kHz} \mathrm{cm}^{-2}$ for both the TPC and the MCH after LS2 [1].

Single Event Latchup (SEL) forms an abnormal high-current state in the CMOS device which can lead to catastrophic failure from excessive heating in the active region of the device, metallization, or bond wires. This may result in the permanent damage of the CMOS device. Therefore, SEL is always considered as a major concern with high severity level for the LHC electronics.

\section{SAMPA V2 Heavy-ion SEL campaign}

It was previously reported that the SAMPA V2 prototypes were susceptible to the high energy proton induced SEL events [3]. The measured SEL cross section for SAMPA V2 was $3.1 \times 10^{-11} \mathrm{~cm}^{2}$ per device. Considering worst HEH flux locations and the number of SAMPA chips for both the TPC and the MCH sub-detectors in the ALICE experiment, the expected Mean Time Between Failure (MTBF) for SEL events were 10 minutes and 5 minutes, respectively.

In May 2017, a heavy-ion irradiation campaign was conducted on SAMPA V2 prototypes at the Universitè Catholique de Louvain (UCL) in Belgium. The purpose of this campaign was to fully characterize the SEL sensitivity for the SAMPA V2 prototypes by determining the energy and ion dependency of the SEL cross-section and to find the source of SEL events. The test setup for the irradiation campaign at UCL is illustrated in Figure 1a where both the SAMPA carrier board and FPGA-based DAQ board were placed inside the vacuum chamber. Communication with the boards was done through custom made cables, which were connected to a programmable power supply and the current controlling system outside the chamber.

The SAMPA V2 irradiation measurements were accomplished with different beams of ions with a broad range of Linear Energy Transfer (LET) from 3.3 to $32.4 \mathrm{MeV} \mathrm{cm}^{2} \mathrm{mg}^{-1}$ presented in the table in Figure 1b. The particles flux was varied from $5 \times 10^{1}$ to $1.5 \times 10^{4}$ particles $\mathrm{cm}^{-2} \mathrm{~s}^{-1}$ with respect to the LET values of the ions, the number of SEL events were counted for each LET value, and a SEL cross-section curve is extracted, as presented in Figure 1b. The points are fitted with a 


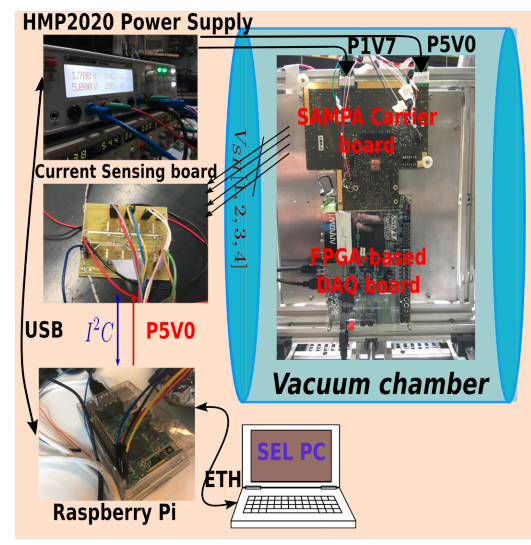

(a) Test setup at UCL.

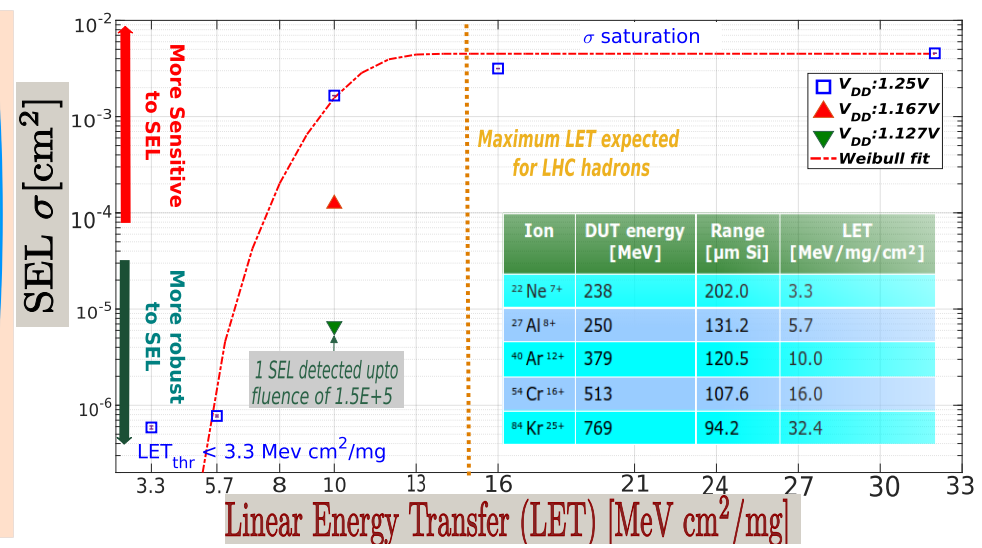

(b) SEL cross-section as a function of the LET values.

Figure 1: SAMPA V2 SEL test setup and results from UCL.

Weibull function, that is commonly used to model this quantity. The extracted results demonstrate that SEL cross-section curve saturates between $10-32.4 \mathrm{MeV} \mathrm{cm}^{2} \mathrm{mg}^{-1}$ LET range, and falls off rapidly between the LET range of 5.7 and $10 \mathrm{MeV} \mathrm{cm} \mathrm{mg}^{-1}$. In contrast, the point at a LET value of $3.3 \mathrm{MeV} \mathrm{cm}^{2} \mathrm{mg}^{-1}$ does not fit the expected fall-off from the Weibull fit, which believes to be due to the indirect energy deposition events, as compared to the direct ionization mechanism from the ions with larger LET values [4]. At a LET value of $10 \mathrm{MeV} \mathrm{cm}^{2} \mathrm{mg}^{-1}$, the reduction of power supply voltage $\mathrm{V}_{\mathrm{DD}}$ confirmed a linear correlation with the SEL sensitivity of the chip, as indicated in the plot.

\section{Experimental methods to localize the origin of SEL in SAMPA V2 digital core}

Heavy-ion tests indicated current jumps (SEL events) only in the digital power domain. The spikes in the digital current domain were correlated with the burst of SEUs observed in the pedestal SRAM IPs. It is worth noting that in the SAMPA V2 prototypes, the pedestal SRAM IPs were single port whereas the other SRAMS IPs were dual port. Two different experimental methods were adopted to inspect the SEL sensitivity for SAMPA V2 memory IPs, as shown in Figure 2.

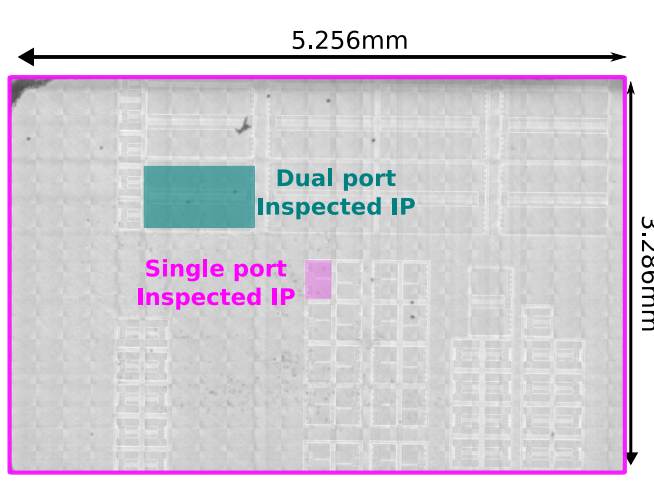

(a) Backside IR camera visualization of SAMPA V2 through silicon substrate during laser campaign.

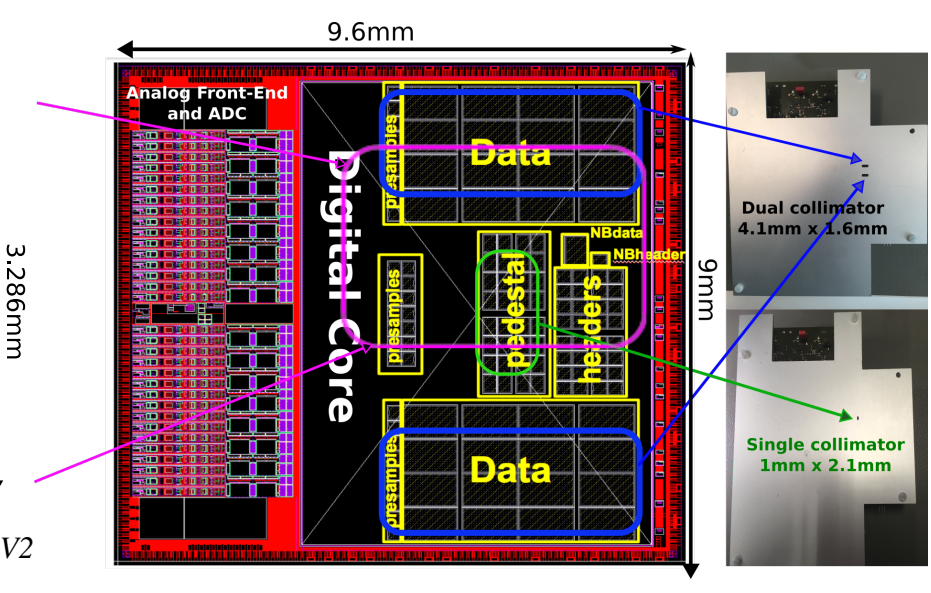

(b) Topside accessible SRAM IPs with collimators during H-I campaign.

Figure 2: Experimental methods to localize the source of SEL events in SAMPA V2. 


\subsection{Heavy-ion tests with collimators}

Two aluminium collimators of thickness $2 \mathrm{~mm}$ were prepared and are indicated in Figure $2 \mathrm{~b}$. The dual collimator had two rectangular openings of $4.1 \times 1.6 \mathrm{~mm}^{2}$ which were sufficient to irradiate $80 \%$ of the dual port SRAM IPs in the V2 digital core. Another single collimator had an opening of $1 \times 2.1 \mathrm{~mm}^{2}$ to irradiate $70 \%$ of the single port SRAM IPs in the V2 digital core. A lot of current spikes were detected in the digital power domain using the single collimator. At the same LET value of $10 \mathrm{MeV} \mathrm{cm}^{2} \mathrm{mg}^{-1}$, no current spike was detected using the dual collimator.

\subsection{Pulsed laser backside irradiation}

In January 2018, a pulsed-laser [5] campaign with PULSCAN system was conducted at IES (Institute of Electronics and Systems) in Montpellier. The laser has a pulse duration of $30 \mathrm{ps,} \mathrm{wavelength}$ of $1064 \mathrm{~nm}$ and spot size of $1.2 \mu \mathrm{m}$, which makes it compatible for SEE testing in semiconductor devices [5]. As presented in Figure 2a, special carrier boards were designed to access the sensitive regions of the SAMPA chips from the backside through the silicon substrate, since the laser source can not penetrate through several metal layers on the top side of the chip.

After determining the laser energy threshold between $117 \mathrm{pJ}$ and $124 \mathrm{pJ}$ for SEL events on the single port memory region, automated scans were performed on an area of $\sim 40 \times 21 \mu \mathrm{m}^{2}$ inside both single and dual port memory arrays. During the scan, the laser was injected with a frequency of $10 \mathrm{~Hz}$ at an $\mathrm{x}-\mathrm{y}$ interval of $0.5 \mu \mathrm{m}$. At each position, the current monitoring system checked for SEL events in real time, and sent a trigger signal to an oscilloscope. Further, the laser system acquired the waveform from the oscilloscope in order to know whether a SEL event occurred. At the end of the scan, the laser system built a sensitivity map ( $\mathrm{x}-\mathrm{y}$ coordinates of the SEL events), and superimposed the map on the actual layout of the chip.

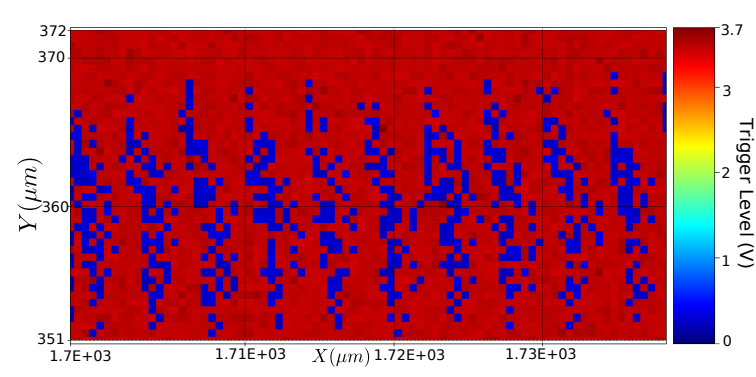

(a) Single port memory with 131 pJ of laser energy.

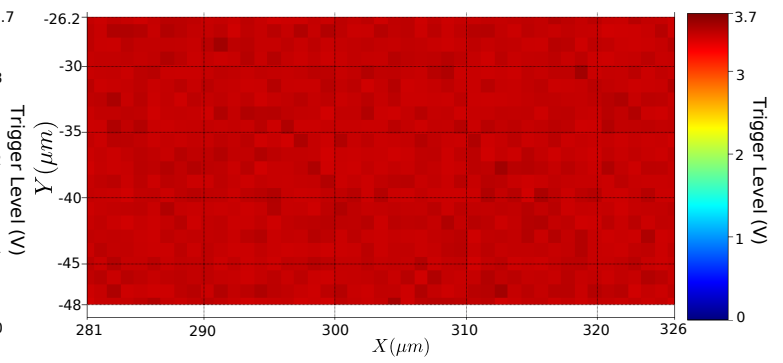

(b) Dual port memory with 690 pJ of laser energy.

Figure 3: SEL sensitive areas during the automated laser scan on SAMPA V2 SRAM IPs.

The results from both laser scans are presented in Figure 3, where the $\mathrm{x}$ and y axes represent the coordinates inside both SRAM IPs, and the colour represents the trigger level. By default, the trigger signal was high (red points), and in case of SEL detection, the trigger signal changed from high to low (blue points). The blue points in Figure 3a indicate all the SEL sensitive regions inside the scanned area of the single port IP. A repetitive pattern is observed for the SEL sensitive regions inside the single port memory array which is probably due to the matrix architecture of the memory array. This is believed to be due to the significantly larger distance between the $n$-well and substrate contacts to the $\mathrm{P}+$ and $\mathrm{N}+$ implants in the memory cells [6]. During the laser scan on the dual port memory array, the trigger signal was constantly high, concluding that no SEL events were detected as presented in Figure $3 b$. 


\section{SEL mitigation in SAMPA V3 and V4}

In the final versions (V3 and V4) of the SAMPA chip, single port IPs were replaced with dual port IPs since both experimental methods verified that dual port IPs were completely robust against SEL effects. From a radiation sensitivity perspective, the functionality of SAMPA V3 and V4 prototype was identical. Another heavy-ion campaign was conducted in November 2017 at UCL to evaluate the SEL sensitivity in the final versions of the SAMPA chip.

\begin{tabular}{|c|c|c|c|c|}
\hline Sample & LET $\left[\mathrm{MeV} \mathbf{~ c m}^{2} \mathbf{~ m g}^{-1}\right]$ & Fluence [particles $\left.\mathbf{~ c m}^{-2}\right]$ & Temperature $\left[{ }^{\circ} \mathbf{C}\right]$ & SEL \\
\hline V4_1 & Scanned at different LET values up to 125 & $1 \times 10^{7} @ 125 \mathrm{MeV} \mathrm{cm}^{2} \mathrm{mg}^{-1}$ & 45 & No \\
\hline V3_1 & 125 & $1.5 \times 10^{7}$ & 60 & No \\
\hline V4_2 & 125 & $1 \times 10^{7}$ & 85 & No \\
\hline
\end{tabular}

Table 1: Summary of SEL heavy-ion irradiation campaign on SAMPA V3 and V4 prototypes.

A short summary of the campaign is presented in Table 1 which concludes that no SEL events were detected on any of the irradiated samples during the campaign. Additionally, during the pulsed-laser campaign, an automated scan was performed on the same pedestal memory region in a SAMPA V4 sample. No SEL events were detected up to $1025 \mathrm{pJ}$ of laser energy which was almost 10 times the energy with which SEL was detected in V2.

\section{Conclusions}

The heavy-ion campaign indicated that SAMPA V2 prototype was highly sensitive to SEL events with a threshold value lower than LET of $3.3 \mathrm{MeV} \mathrm{cm}^{2} \mathrm{mg}^{-1}$. Both the heavy-Ion and pulsed-laser campaigns confirmed that the cause of SEL events was related to single port SRAM IPs in the V2 prototypes. Pulsed-laser automated scan verified that the SEL sensitive areas were located inside the memory cells of the single port SRAM IP and not in the periphery circuits. The final campaign assured that the final versions of the SAMPA chip are not susceptible to SEL effects and fully qualify to operate in the ALICE radiation environment.

\section{Acknowledgement}

The authors acknowledge all the people that have participated in the SAMPA project. This work is partially funded by Norwegian PhD Network on Nanotechnology for Microsystems, which is sponsored by the Research Council of Norway, Division of Science, under contract no. 221860/F60.

\section{References}

[1] P. Antonioli et al. [ALICE Collaboration], Technical design report for the upgrade of the ALICE Readout \& Trigger System, CERN-LHCC-2013-019, [ALICE-TDR-015].

[2] J. Adolfsson et al., SAMPA Chip: the New 32 Channels ASIC for the ALICE TPC and MCH Upgrades, JINST, 12 (2017) C04088.

[3] S.M. Mahmood et al., First irradiation test results of the ALICE SAMPA ASIC, Proceedings of Science (POS). TWEPP, 17 (2018) 093.

[4] R.G. Alia et al., Proton Dominance of Sub-LET Threshold GCR SEE Rate, IEEE Transactions on Nuclear Science, 64.1 (2017).

[5] Buchner, Stephen P et al., Pulsed-laser testing for single-event effects investigations, IEEE Transactions on Nuclear Science, 60.3 (2013) 1852-1875.

[6] Puchner, H., et al., Elimination of single event latchup in 90nm SRAM technologies, 2006 IEEE International Reliability Physics Symposium Proceedings. 